\section{P4.062 GROUP SEX, DRUGS, AND INTERNET USE AMONG MEN WHO HAVE SEX WITH MEN (MSM) IN BANGKOK, THAILAND}

doi:10.1136/sextrans-2013-051184.0960

'W Thienkrua, ${ }^{2,1,3} \mathrm{C}$ S Todd, ${ }^{1} \mathrm{~A}$ Varangrat, 'T Chemnasiri, 'J Tongtoyai, 'P Luechai, ${ }^{1} \mathrm{~K}$ Pancharoen, ${ }^{1} \mathrm{~A}$ Sriporn, ${ }^{4} \mathrm{~A}$ Chitwarakorn, ${ }^{1,5} \mathrm{~T}$ H Holtz. ${ }^{1} T$ Thailand Ministry of Public Health - U.S. Centers for Disease Control and Prevention Collaboration, Nonthaburi, Thailand, 'FHI360, Bangkok, Thailand, '3AVRAM Corporation, Miami, FL, United States, ${ }^{4}$ Thailand Ministry of Public Health, Nonthaburi, Thailand, ${ }^{5}$ Centers for Disease Control and Prevention, Atlanta, GA, United States

Background In several urban areas globally, reports describe increasing sexually transmitted infection (STI) prevalence attributed to group sex characterised by Internet-based networks and drug use among men who have sex with men (MSM). In Thailand, "high parties" (HPs) (group sex with concomitant drug use) have emerged as possible drivers of STI epidemics among MSM; this analysis aims to describe associated factors.

Methods In the ongoing Bangkok MSM Cohort Study, participants are Thai MSM aged > 18 years followed every 4 months for 60 months for HIV testing and behavioural questionnaire completion. Questions regarding HPs were introduced in April 2010, with analysis limited to men contributing HP data through January 2013. We assessed HP descriptors among attendees using proportions and factors associated with HP attendance using generalised estimating equations.

Results Of 1340 men, prior HP attendance was reported by 207 (15.4\%), of whom 161 joined in the previous 4 months. Of recent attendees, $60.3 \%$ reported consistent condom use, while $11.7 \%$ reported never using condoms at HPs. In a multivariable model, HP attendance in the previous 4 months was independently associated with sex while intoxicated (Adjusted Odds Ratio (AOR) 7.60, 95\% CI: 4.43-13.0), methamphetamine (AOR 7.10, 95\% CI: 4.15-12.1), gamma hydroxybutyrate (AOR 3.08, 95\% CI: 1.15-8.20) and Viagra (AOR 2.35, 95\% CI: 1.60-3.43) use, finding partners in Internet chat rooms (AOR 1.56, 95\% CI: 1.12-2.27), and sex with casual partners at school/work (AOR 2.61, 95\% CI: 1.05-6.53), in a partner's home (AOR 1.66, 95\% CI: 1.20-2.29), or in a hotel (AOR 1.56, 95\% CI: $1.12-2.17$ ) in the previous four months.

Conclusions HP attendance is strongly associated with multidrug use and sex while intoxicated, increasing STI transmission risk. Internet-based education regarding drug use and condoms and non-coitally dependent prevention technologies are urgently needed among MSM in Bangkok.

\section{P4.063 CORRELATES OF VOLUNTARY HIV TESTING AND COLLECTING TEST RESULTS AMONG MEN WHO HAVE SEX WITH MEN IN INDIA}

doi:10.1136/sextrans-2013-051184.0961

'K Nagarajan, ${ }^{2} \mathrm{~S}$ Ramanathan, ${ }^{1} \mathrm{M}$ K Mainkar, ${ }^{2} \mathrm{~L}$ Ramakrishnan, ${ }^{1} \mathrm{R}$ S Paranjape. 'National AIDS Research Institute, Pune, India; ${ }^{2}$ FHI 360, New Delhi, India

Background As HIV prevention programmes in India prioritise voluntary HIV counselling and testing, among high-risk groups, hidden populations like Men Who Have Sex with Men (MSM) face barriers in accessing these services. Previous studies among MSM have focused on testing behaviours and rarely considered if test results were obtained. To inform prevention interventions we examined factors facilitating both voluntary HIV testing and collecting test results (VT\&CTR) in two high HIV prevalent states in India.

Methods Data for the current analysis were drawn from a large scale bio-behavioural surveys conducted among MSM between 2009-2010 in Tamil Nadu (TN) (n-1757) and Maharashtra (MH) (n-692), India. A composite variable for VT\& CTR was created and chi-square test, multiple logistic regression analysis adjusted for socio-demographic and contextual factors were used to assess the correlates of VT\& CTR.

Results Of the total, nearly half have undertaken VT\&CTR in $\mathrm{MH}$ (48.3\%) and TN (57.8\%). Bivariate analysis in $\mathrm{MH}$ indicates, "early sexual debut" distinguished MSM who opted for (37.6\%) VT\&CTR from MSM who did not (23\%). In the multivariate analysis, factors associated with increased odds for VT\& CTR were: having received HIV/STI information from peers in MH (AOR-4.3 CI-1.7-10.5) \& TN (AOR 5 CI 3.5 1.0-6.5) and knowing AIDS can lead to death in TN (AOR 2.5 CI-2.3-10.8) and MH (AOR-1.5 CI-1 -2.3). Knowledge on HIV transmission (TN: AOR 1.5, CI-1.0-2.3), HIV risk perception (MH: AOR 2.8, CI-1.2-6.3) consistent condom use with regular male/hijra partners (TN:AOR 2.2, CI-1.5-3.3) and female partners (MH: AOR 4.4 CI 1.1-16) were additional factors associated with VT\&CTR. However, sexual-identity, collective membership and past STI symptom were not associated with VT\&CTR.

Conclusion Findings indicate that HIV prevention efforts can be successful in increasing voluntary testing behaviours among MSM Strengthening the existing behavioural interventions and providing tailored strategies can increase voluntary testing beyond the current levels.

\section{P4.064 STATUS OF STIS AMONG MSMS AND TRANSGENDERS IN THE EASTERN REGION OF NEPAL}

doi:10.1136/sextrans-2013-051184.0962

N Lamichhane. Koshi Health and Science Campus, Biratnagar, Nepal

Background Sexually transmitted infections are the major problems among most high risk population of HIV and AIDS in Nepal. Studies were showed Men having sex with men and transgender has higher risk of HIV and STI in the eastern of Nepal.

Methods The cross-sectional descriptive studies conducted among $156 \mathrm{MSM}$ and transgender in the eastern region of Nepal. The clinical examination and syndromic informations were taken through checklist and questionnaire was asked to assess the risk behaviour The verbal consent was taken from the respondents.

Results Fifty six percent of respondents didn't use condom and lubricants during their sexual intercourses. One quarter of respondents didn't hear about lubricants. Fifteen percent of them did sexual contact after taking alcohol and most of them didn't care about their correct process of condom application. Twenty percent of respondents have more than two sexual partners which they had to involve in sexual intercourse at least two times in the months. The use and non use of condom is associated with the STI incidence $(\mathrm{P}=0.001, \alpha=5)$. Among the total respondents forty percent had at least one symptoms of STI such as urethral and anal discharge, genital wart, itching and ulceration. Most of the respondents feared to go to the doctors because of social stigma.

Conclusion STI among MSM and Transgender was higher. The social acceptance was low. The MSM and Transgenders should encourage attending STI clinics for their sexual problems remedies.

\section{P4.065 HIGH RATES OF NON-CONSENSUAL SEX ANS ASSOCIATION WITH SEXUAL HEALTH AMONG A LARGE NATIONAL SAMPLE OF YOUNG PEOPLE IN THE NETHERLANDS}

doi:10.1136/sextrans-2013-051184.0963

${ }^{1}$ C Somers, ${ }^{1,2}$ N H T M Dukers-Muijrers, ${ }^{3} \mathrm{H}$ de Graaf, ${ }^{4} \mathrm{~S}$ Meijer, ${ }^{1,2} \mathrm{~A}$ Niekamp,${ }^{1,2} \mathrm{C}$ J P A Hoebe. 'Department of Sexual Health, Infectious Diseases and Environmental Health, South Limburg Public Health Service, Geleen, The Netherlands; ${ }^{2}$ Department of Medical Microbiology, School of Public Health and Primary Care (CAPHRI), Maastricht University Medical Centre (MUMC+), Maastricht, The Netherlands; ${ }^{3}$ Research Department, Rutgers WPF, Utrecht, The Netherlands; ${ }^{4}$ Youth Department, STI AIDS The Netherlands, Amsterdam, The Netherlands 